\title{
QoESoft: QoE Management Architecture for Softwarized 5G Networks
}

\author{
Alcardo Alex Barakabitze, Madhusanka Liyanage and Andrew Hines \\ School of Computer Science, University College Dublin, Ireland \\ \{alcardo.barakabitze,madhusanka,andrew.hines\}@ucd.ie
}

\begin{abstract}
For 5G network softwarization, Software Defined Networking (SDN) and Network Function Virtualization (NFV) have emerged as key technologies to enable efficient network management. 5G network virtualization will allow multiple service providers to share a single physical infrastructure offering an abstraction between the user and physical resources. As we move towards softwarized 5G networks, scalability issues, network performance with respect to Quality of Service $(\mathrm{QoS})$ and Quality of Experience (QoE) and QoE-aware resource management remain less studied topics. In this paper, we adopt the SDN control logic to perform an autonomic QoE-aware resource management approach in virtualized $5 \mathrm{G}$ networks. We propose QoESoft, a network softwarization strategy for multimedia streaming services that performs a dynamic link and switch resources management in softwarized 5G systems. With QoESoft, we aim to improve user QoE for multimedia services through efficient monitoring and adaptation of the average load of the substrate links and switches and resource costs of mapped virtual links in 5G networks. The performance of the proposed approach is analysed in testbed environments. Preliminary results indicate that, QoESoft achieves better performance than the compared baseline approach in terms of link and switch resources utilization by $78 \%$ and 70\% respectively. A video reception quality ratio metric and visual inspection on the output video both confirmed significant improvements in video QoE.

Keywords-SDN, Quality of Experience (QoE), 5G, QoE, Multimedia Streaming Services, NFV. QoE Management, Video Quality
\end{abstract}

\section{INTRODUCTION}

The concepts underlying the development of the future mobile/wireless networks have been explored in recent years with a vision towards $5 \mathrm{G}$ systems [1]. 5G network softwarization using Software Defined Networking (SDN) and Network Function Virtualization (NFV) will provide the flexibility needed by service providers, mobile network operators in adapting the future dynamic market drivers (e.g., multimedia streaming services - such as $\left.4 \mathrm{~K} / 8 \mathrm{~K} / 12 \mathrm{~K}, 360^{\circ}\right)$ [1], [2].

NFV and SDN promise to steer the 'Softwarization' of future telecommunication [1] systems. This will radically change the design of $5 \mathrm{G}$ networks and offer flexibility, reconfigurability and programmability to support variety of use cases such as multimedia streaming services. NFV in $5 \mathrm{G}$ will promote the timely delivery of an End-to-End (E2E) services, reduce capital expenditure (CAPEX) and operating expenditure (OPEX), and enable network functions to be deployed dynamically. One or multiple service providers in $5 \mathrm{G}$ networks will be able to create Virtual Networks (VNs) and provide multimedia streaming services to the end-users with high QoE by using Substrate
Networks (SNs). The SNs can be managed and controlled by $5 \mathrm{G}$ Infrastructure Providers (InPs). However, as InPs can host multiple VNs and support their complete isolation, an outstanding challenge is how to allocate and manage resources efficiently to multiple VNs. Traffic and network control is one of the $\mathrm{InP}$ requirements in softwarized $5 \mathrm{G}$ networks.

SDN [2] has emerged as one of key technologies in softwarized 5G systems where the control logic of each VN can run on the SDN controller rather than on physical switches [3]. Using SDN, each virtual link can be represented as a flow and therefore be able to define a $\mathrm{VN}$ as a set of flow rules in different switches [4]. However when a virtual flow is split into multiple subflows in SDN environments, each subflow requires new flow rules in each of the OpenFlow switches along the substrate path. SDN switches store flow rules in a Ternary Content-Addressable Memory (TCAM) which is power hungry and practically supports a limited size of $2 \mathrm{k}-20 \mathrm{k}$ forwarding rules [5], [6]. As stated in [5], a larger number of forwarding flow rules in softwarized 5G network, along each substrate delivery path, would increase the load at the controller and SDN switches. 5G networks need to support and deliver high video quality and sustained multimedia streaming services (e.g., $360^{\circ}$ videos, video gaming, $4 \mathrm{~K} / 8 \mathrm{~K} / 12 \mathrm{~K}$ ). Switch memory to support streaming services is expensive and power hungry [6] so giving management considerations to both link and switch resources is necessary in softwarized $5 \mathrm{G}$ networks. For such an approach, a Substrate Network Provider (SNP) could provide a common substrate to support a number of virtual $5 \mathrm{G}$ networks. These VNs, implemented by virtual nodes connected through virtual links, in turn would provide a range of different services including multimedia streaming services in softwarized $5 \mathrm{G}$ networks.

In this paper, we extend the QoE-softwarized architecture for 5G that we introduced in [2] and propose QoESoft, a network softwarization approach for multimedia streaming services that performs a dynamic link and switch resources management in softwarized 5G networks. We extend the idea introduced in [2] and perform real-time video streaming experiments using Mininet and POX controller. Different from other approaches that only consider link resources (e.g., bandwidth) [7], [8], our approach takes into account the $\mathrm{VN}$ resource costs as well as node resources (memory). We implement a QoE-sdnFlow manager and QoE-sdnFlow monitor entities as an extension to the POX controller [5] for managing the end-user's QoE 
and monitoring the average load of the substrate links and switches and resource costs of mapped virtual links. The $Q o E$ sdnFlow manager is able to modify (adds and/or deletes) flow rules in switches using the information provided by the arrivals and departures of VN requests during video streaming. Note that, the VN requests arrive and depart in a dynamic manner. Such a dynamic QoE-aware resource management leads to better resource utilization efficiency that translates to delivering multimedia services in 5G systems with high QoE [9].

Due to the dynamic arrival of $\mathrm{VN}$ requests, some virtual flows would utilize more resources during the mapping process of VN nodes and links to SN nodes and links. However, when some VNs migrate, more efficient flows can be established leading to an efficient delivery of high quality services to end-users. Our proposal seeks to empower mobile operators and SPs who are eager to deliver quality multimedia services through an autonomic software life-cycle management approach in 5G systems. Our innovative approach overcomes the key QoE provisioning limitations in traditional networks such as (1) network management complexity, (2) lack of fine-grain control of networks and suboptimal utilization of resources, (3) static nature of current networks (which are unable to dynamically adapt to changing multimedia services, network transmission/traffic or end-user's demand) and (4) manual configuration and provisioning of many QoS tools. The contributions of this paper are three-fold:

- We present a dynamic QoE-aware resource management mechanism "QoESoft" for future multimedia streaming services in softwarized $5 \mathrm{G}$ networks.

- We implement a QoE-sdnFlow manager and $Q o E$ sdnFlow monitor entities introduced in our previous work [2]. These entities are extended to the POX controller for monitoring and managing the average load of the substrate links and switches and resource costs of mapped virtual links in $5 \mathrm{G}$ networks.

- We present preliminary results to show the credibility and innovation of the "QoESoft" approach through experiments based on Dynamic Adaptive Video Streaming over HTTP (DASH).

The rest of this paper is organized as follows: we describe related works in section II. The resource management problem in SDN-based virtualized 5G networks is described in section III. We introduce the QoE-aware dynamic resource management proposal in section IV. Section V presents the network model and a QoE-aware resource management algorithm for softwarized $5 \mathrm{G}$ networks while section VI presents the performance and evaluation of our proposal. Finally, section VII concludes the paper.

\section{RelATED WORK}

The QoE management of future multimedia services in SDN-based virtualized networks for $5 \mathrm{G}$ is an active research topic [2], [3], [10]. The reactive $\mathrm{VN}$ resource management techniques for dynamic resource management in virtualized networks are presented in [7]. To control migration costs, the
VN reconfiguration scheme migrates a single/multiple node(s) and its associated $\mathrm{VN}$ links in a condition of a failed mapping but without considering the switch loading. A selective VN reconfiguration approach that prioritizes the reconfiguration for overloaded VNs is presented in [8]. Feng et al. [11] propose a fair allocation and a price-based joint allocation model in SDN. The proposed approach can guarantee the fairness of network resource allocation and minimize the mean delay time. The resource management mechanism in the SDN control is proposed in [4] where the mapping of VN to $\mathrm{SN}$ is dynamically achieved based on network status. However these works do not consider the QoE aspects in the context of 5G networks. Barakabitze et al. [2] introduce a QoE-softwarized architecture for managing future multimedia services in $5 \mathrm{G}$ networks using SDN and NFV. Different SDN strategies regarding traffic predictions, load balancing, radio resource allocation and user density predictions in 5G networks are analysed in [3]. We note also that these works do not consider the QoE-aware resource management of link and switch and the cost of $\mathrm{VN}$ resources in softwarized 5G networks. Our aim is to improve the QoE of the delivered multimedia streaming services through efficient and flexible mechanisms for controlling and managing all the available network resources in a softwarized 5G systems. To the best of our knowledge, this paper is the first attempt to study the dynamic resource management in softwarized 5G networks by taking into account the end-user's QoE.

\section{PROBlem Formulation AND DESCRIPTION}

\section{A. E2E $5 G$ network video streaming use case}

Fig. 1 illustrates an E2E 5G network video streaming use case scenario on an SDN/NFV network. A chain of VNFs provide supports to the network bandwidth and high quality expectations from users for different multimedia streaming services (e.g., Video on Demand (VoD)). The user's video request is transported across these Virtual Network Functions (VNFs). Depending on the user's request, the VNFs formulate a service graph to establish the VNFs Fowarding Graph (VNFFG). The VNF-FG shows the graph of logical links connecting VNF nodes for the purpose of describing the traffic flow between these VNFs. The VNF-FG is stored in the VNFs' database (DB). There remains a challenge to manage link and switch resources for virtual $5 \mathrm{G}$ networks that are mapped to substrate $5 \mathrm{G}$ networks with consideration towards maintaining the user's QoE.

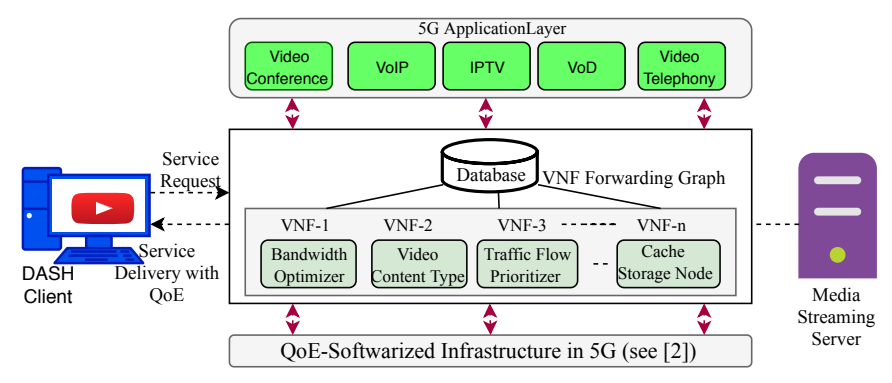

Fig. 1: End-to-end 5G multimedia streaming use case scenario 


\section{B. Virtual Network Assignment Problem Description}

We describe the virtual to substrate network mapping problem using an example of a network shown in Fig. 2. Consider the virtual network $A B C$ that is to be mapped onto substrate SDN network $\{\mathrm{S} 1 \rightarrow \mathrm{S} 2 \rightarrow \mathrm{S} 3 \rightarrow \mathrm{S} 4 \rightarrow \mathrm{S} 5 \rightarrow \mathrm{S} 6\}$. To achieve this, each virtual switch $i \in(A, B, C)$ is mapped to the substrate SDN OpenFlow switch $m \in\{\mathrm{S} 1 \rightarrow \mathrm{S} 2 \rightarrow \mathrm{S} 3 \rightarrow \mathrm{S} 4 \rightarrow \mathrm{S} 5 \rightarrow \mathrm{S} 6\}$ with enough memory and connected links of sufficient bandwidth to support the virtual links. This mapping is made possible and accomplished using the node and link assignment as explained in the next subsections. However, such an assign-

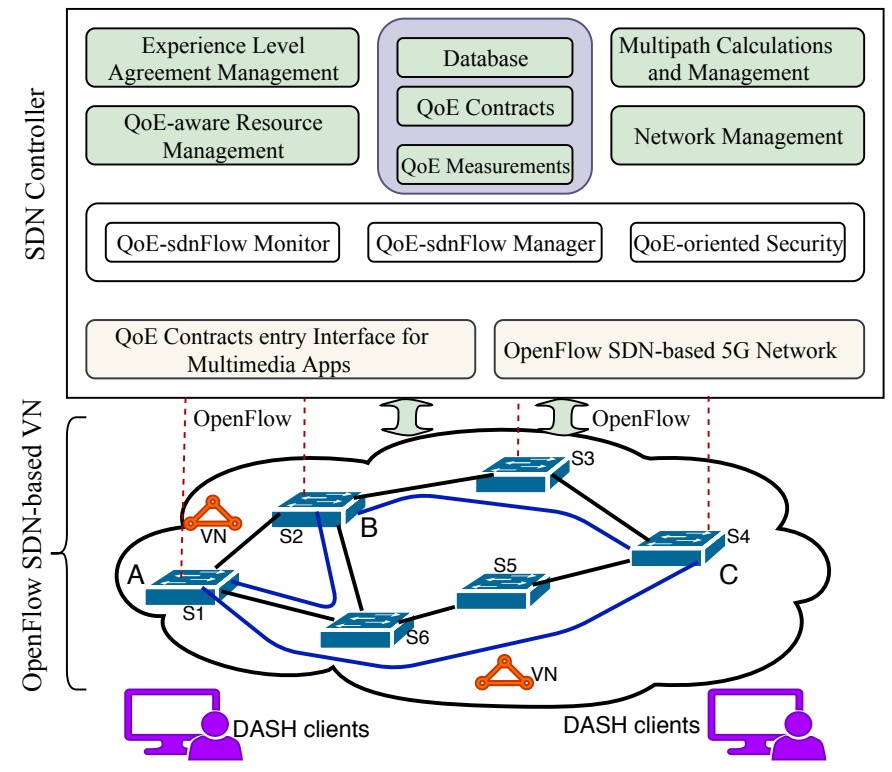

Fig. 2: Proposed QoE-aware dynamic resources management in softwarized $5 \mathrm{G}$ networks

ment of virtual nodes and links to the corresponding substrate nodes and links would cause problems to a softwarized $5 \mathrm{G}$ network. Consider an assignment of virtual links $A B$ and $A C$ to substrate paths $\{\mathrm{S} 1 \rightarrow \mathrm{S} 6 \rightarrow \mathrm{S} 2\}$ and $\{\mathrm{S} 1 \rightarrow \mathrm{S} 6 \rightarrow \mathrm{S} 4\}$ respectively with substrate switch $S 6$ being the bridging point of multimedia flows. Such an assignment requires the VN layer to define flow rules in virtual switch $A$ to direct multimedia traffic to switches $B$ and $C$ respectively. To ensure the forwarding flow rules arrive to their intended destination, extra rules would be needed at the physical $5 \mathrm{G}$ network layer for each of the flows in a switch. The number of flow rules would increase further when path splitting [8] techniques are used for virtual link mapping. One of the visions of $5 \mathrm{G}$ systems is to enable logical network slices that will support and meet the specific demands of use cases such as video streaming and personalized $\mathrm{TV}$, by making use of big data analytics to shape traffic for video QoE. Having a high number of rules for a single flow in $5 \mathrm{G}$ network would result in $5 \mathrm{G} \mathrm{VN}$ owners incurring high costs for their network slices. It may also cause the requests of $5 \mathrm{G}$ VN to be rejected due to the depletion of SN switch memory. Having a balanced number of forwarding flow rules across the $5 \mathrm{G} \mathrm{SN}$ is essential to avoid over/under utilization of available resources and reduce congestion. This would in-turn result in an improved QoE of transmitted multimedia streaming services to the end-users. To this end, a dynamic link and switch QoEaware resources management in a softwarized $5 \mathrm{G}$ network is necessary to ensure that end-users receive multimedia services with an improved QoE. A QoE-aware dynamic and demandbased resource allocation is seen as one possibility of improving the user's perceived QoE by allocating more network resources to specific video flows or by changing the forwarding behavior of switches.

\section{QOE-AWARE RESOURCE MANAGEMENT SYSTEM IN SOFTWARIZED 5G NETWORKS}

This section introduces QoESoft, a QoE-aware resource management system in an SDN-based VN for $5 \mathrm{G}$ systems as shown in Fig. 2. Our proposal includes the QoE-sdnFlow manager, QoE-sdnFlow monitor modules and a database. These modules are implemented as an extension to an existing POX SDN controller. It also consists of an Experience Level Agreements (ELA) [12] module that signifies a well-defined SLA, mechanisms that focus on the end-user's QoE. The ELA are introduced to provide new ways of QoE-based business contracts between service providers and users. The focus of ELA is on 5G-Quality of Business (5GQoBiz) [9] and QoE-based multimedia streaming service provisioning to the end-users. We provide details of the QoE-aware resource management 5G architecture components as follows.

\section{A. QoE-sdn Flow Monitor}

The QoE-sdnFlow monitor is used to monitor the resource utilisation of mapped virtual link (both link and switch) and the average loading of the substrate links and switches. This entity also performs the QoE measurements and QoE-estimation per multimedia traffic flow. Based on the identified multimedia traffic type such as (e.g., video/voice), the QoE-sdnFlow monitor selects the QoE estimation/assessment model available for estimating the QoE. It can also formulate the per-client network resource requirements (e.g., QoS) and map them to the optimal per-client $\mathrm{QoE}$ using an exponential mapping function shown below.

$$
M_{\text {function }}=\left\{\begin{array}{l}
Q o S=K \times(W \times B W) \\
Q o E=\alpha \times e^{-}(\beta \times Q o S)+\gamma
\end{array}\right.
$$

$W$ is a weight factor that indicates the degree of QoS metric for different applications and services. For example, bandwidth can be the $W$ for DASH video services. $K$ is a constant access network factor while $B W$ is the bandwidth. $\gamma$ is a video codec parameter. $\alpha$ and $\beta$ are the parameters on the network level for QoS/QoE service differentiation and personalization (for gold, silver and bronze users) in softwarized 5G systems.

\section{B. QoE-sdnFlow Manager}

The QoE-sdnFlow manager is set to acquire network topology information after successful VN to SN mapping and implement QoE based network policies and QoE-aware resource 
allocation. It performs the overall customer's video streaming experience of multimedia services and enable a QoE-aware network resources management for softwarized $5 \mathrm{G}$ systems. It further enhance the quality of business through a well-defined QoE contracts between service providers, operators and end users. It is worth mentioning that, the QoE-sdnFlow manager is a state-action oriented entity of which an action on forwarding flow rules from the SDN switches is taken based on a specific state.

1) Actions on Flow Rules: We define action as an operation that is used for forwarding the video packet to a port or an operation that modifies the transmission of a video packet to a different path. Addition, modification and deletion of forwarding flow rules are the three possible actions which can happen in softwarized switches.

a) Flow Rule Addition: The QoE-sdnFlow manager is responsible for adding forwarding flow rules to the corresponding switches for all the virtual links after successful VN to SN mapping. With reference to Fig. 2, the SDN controller would add rules to switches $S 1, S 6$, and $S 2$ to establish the substrate path $\{\mathrm{S} 1 \rightarrow \mathrm{S} 6 \rightarrow \mathrm{S} 2\}$ for virtual link $A B$ after successful mapping of VN $A B C$. The flow rules are also added to the database where the present and the past resource utilisation and availability are updated and stored.

b) Flow Rule Deletion: The allocated SN resources are released when the $\mathrm{VN}$ departs or changes its assignment. Therefore, when a virtual link departs or is migrated, some flow rules are deleted by the QoE-sdnFlow manager. For example, when the virtual link $A B$ is migrated from substrate path $\{\mathrm{S} 1 \rightarrow \mathrm{S} 6 \rightarrow \mathrm{S} 4\}$ to $\{\mathrm{S} 1 \rightarrow \mathrm{S} 4\}$, then the flow rule in switch $B$ corresponding to the previous assignment is deleted because of becoming useless.

c) Flow Rule Modification: The departure or a change of a VN assignment for resources in a better substrate path can trigger a modification to a flow rule. For example, when there are available resources on the substrate link $\{\mathrm{S} 1 \rightarrow \mathrm{S} 2\}$, the virtual link $A B$ can be migrated from $\{\mathrm{S} 1 \rightarrow \mathrm{S} 6 \rightarrow \mathrm{S} 2\}$ to $\{\mathrm{S} 1 \rightarrow \mathrm{S} 2\}$ which also causes the flow rule in switch $S 1$ to be modified.

\section{Experience Level Agreement Management and QoE Con- tracts}

To formalize the QoE-oriented contracts between customers and service providers, the proposed resource management approach incorporates the management aspects of ELA. QoEoriented business model that we introduced in our recent work [9] is also integrated in the softwarized 5G architecture in Fig. 2. Such an approach is set to foster new business practices for service providers with a minimum QoE guarantee to their customers. We envision that, mobile network operators and service providers in the $5 \mathrm{G}$ era should focus towards QoE marketization through ELA management and QoE-related contracts, going beyond the current QoS-oriented SLAs.

\section{Network Model and QoE-Aware Resource MANAGEMENT ALgORITHM}

\section{A. Network Model}

The substrate SDN network is modelled as a weighted undirected graph $G_{s}=\left(V_{s}, E_{s}\right)$ where $V_{s}$ and $E_{s}$ represents a set of of SN switches and links respectively. The VN is modelled as the graph $G_{v}^{i}=\left(V_{v}^{i}, E_{v}^{i}\right)$ with a set of virtual nodes $V_{v}^{i}$ and virtual links $E_{v}^{i}$. Each substrate switch $(m \ldots n) \in S$ has a memory $M_{m}$ which is the TCAM [6]. TCAM stores the routing rules given by an SDN controller. The substrate switches $m$ and $n$ are connected by substrate SDN links $l_{m n} \in L$ having a bandwidth of $B_{w}$. The assignment of the $i_{t h} \mathrm{VN}$ is done using the node and link assignment as described below.

1) Node assignment: Each virtual node is assigned to a substrate node formalized as a mapping $f_{N}^{i}: V_{v}^{i} \rightarrow V_{s}$ such that $f_{N}^{i}(v) \in V_{s}, \forall v \in V_{v}^{i}$ and $f_{N}^{i}(u)=f_{N}^{i}(v)$ iff $u=v$.

2) Link assignment: The assignment of each virtual link to a substrate path between the corresponding substrate nodes is formalized using the following mapping: $f_{L}^{i}: E_{v}^{i} \rightarrow P_{s}$ such that $f_{L}^{i}(\overline{u v}) \in P_{s}\left(f_{N}^{i}(u), f_{N}^{i}(v)\right) \forall(\overline{u v}) \in E_{v}^{i}$ where $P_{s}$ is the set of all substrate paths. $\overline{u v}$ is the set of virtual links from virtual nodes $\bar{u}$ to $\bar{v}$.

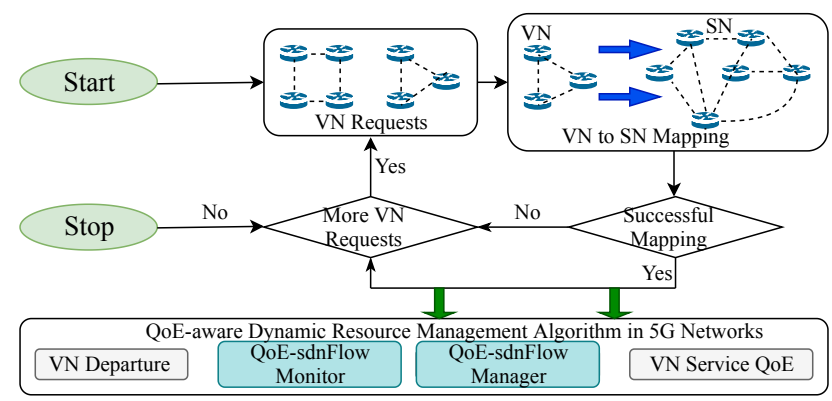

Fig. 3: QoE-aware resource management algorithm in softwarized $5 \mathrm{G}$.

The allocated substrate resources are released when the VN departs. Furthermore, if an existing VN changes its assignment, the substrate network will release previously allocated resources by the old assignment and allocate new network resources based on the new assignment. The total available resources utilisation $R_{u^{\prime} v^{\prime}}$ used by virtual link $l_{u^{\prime} v^{\prime}}$ is defined using equation 2 .

$$
R_{u^{\prime} v^{\prime}}=\alpha \sum_{\left.l_{u v} \in P l_{u^{\prime} v^{\prime}}\right)}\left(B_{u^{\prime} v^{\prime}}\right)+\beta \sum_{u \in P l_{u^{\prime} v^{\prime}}}\left(M_{u^{\prime}}\right)
$$

$P l_{u^{\prime} v^{\prime}}$ indicates the substrate path where the virtual link $l_{u^{\prime} v^{\prime}}$ has been mapped. For each substrate link onto which it is mapped, $B_{u^{\prime} v^{\prime}}$ is the bandwidth used by the video flows of the virtual link. $M_{u^{\prime}}$ is an SDN switch memory used by the forwarding flow rules that is associated with it. It is worth noting that, each virtual links is evaluated to determine the available resources, $R_{u^{\prime} v^{\prime}}$ every time a $\mathrm{VN}$ is mapped onto the SN.

The available substrate resources $A_{u^{\prime} v^{\prime}}$ as shown in equation 3 for each virtual link $l_{u^{\prime} v^{\prime}}$ is defined as the total amount of 
resources available for all switches and links on the substrate path $P l_{u^{\prime} v^{\prime}}$. Priority is given to the substrate links or switches that has less resources especially when decisions has to be made on which virtual links to migrate.

$$
\left.A_{u^{\prime} v^{\prime}}=\alpha \sum_{\left.l_{u v} \in P l_{u^{\prime} v^{\prime}}\right)}\left(B_{u v}-B_{u v}^{\prime}\right)+\beta \sum_{\left.u \in P l_{u^{\prime} v^{\prime}}\right)}\left(M_{u}\right)-M_{u}^{\prime}\right)
$$

where $M_{u}^{\prime}$ indicates the total memory of the substrate switch $u$ that is currently allocated to the virtual switches where it has been mapped. $B_{u v}^{\prime}$ indicates the total bandwidth of the substrate link $l_{u v}$ that is allocated to virtual links. The weights $W_{u^{\prime} v^{\prime}}$ of virtual links in the network are defined by equation 4 .

$$
W_{u^{\prime} v^{\prime}}=\lambda R_{u^{\prime} v^{\prime}}-\mu A_{u^{\prime} v^{\prime}}
$$

where $\lambda$ and $\mu$ are constants that determine the resource utilisation or the availability of resources on the substrate links respectively.

\section{B. QoE-aware Resource Management Algorithm}

Fig. 3 shows the QoE resource management algorithm considered in this paper. We extended the approach proposed in [2] by implementing two important modules for QoE management and resource allocations. The mapping or SN resource assignment to individual nodes and links of $\mathrm{VN}$ is one of the fundamental operations of network virtualization. Specifically, each virtual node is assigned to one of the substrate nodes and each virtual link is assigned to a substrate path that connects the corresponding end nodes.

In the multimedia streaming use case scenario shown in Fig. 1, VN demands can arrive at any time instance and as is the case for requests to setup VNs with different topologies and lifetimes. Upon the arrival of a $\mathrm{VN}$ request, the topology mapping to the SN is performed to achieve low and balanced load on both substrate nodes and links. If the mapping is successful, the dynamic QoE-sdnFlow manager module is triggered, otherwise other VNs' requests are considered. The QoE-sdnFlow manager ensures that, during a given $\mathrm{VN}$ service period, then link remapping/migrations can be done. Such a mechanism increases the substrate resource utilization efficiency that allows the substrate network to accommodate more VNs with limited resources and reduce congestion. The weight $W_{u^{\prime} v^{\prime}}$ for each virtual link $l_{u^{\prime} v^{\prime}}$ is recomputed by the QoE-sdnFlow manager when a previously accepted request departs or the $\mathrm{VN}$ request is accepted.

\section{Vi. Performance Analysis and Evaluation}

\section{A. Experimental Testbed}

To evaluate the credibility of our approach, we extended the POX controller by implementing the QoE-sdnFlow and QoEsdnFlow manager modules that perform the QoE estimation and QoE optimization to enhance the end-user's QoE in 5G networks. The 'Big Buck Bunny' video sequence with video resolution of 1920x1080 pixels and a duration of 9 minutes and 56 seconds was used for video transmission. The video

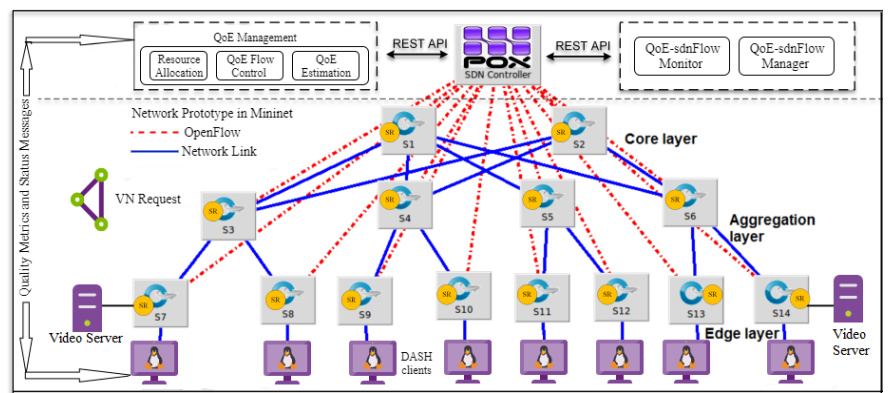

Fig. 4: Experimental testbed for 5G softwarized network

sequence was encoded based on ffmpeg version 3.3.4 with the libx265 and segmented (at 2, 4, 6, 8 and 10 seconds) using the GPAC MP4Box [13] for 3 different video resolutions (360p, 480p, 720p) with video encoding rate of $1.0888 \mathrm{Mbps}$, $1.536 \mathrm{Mbps}$ and $2.496 \mathrm{Mbps}$ respectively. The video segments were then stored in Apache server that is attached to the Mininet prototype running in a virtual machine as shown in Fig. 4. We consider $500 \mathrm{VN}$ arrivals during video streaming and run experiments 20 times from the server to the DASH client. As illustrated in Fig. 4, the topologies for VN requests are generated using Brite [14] while the $\mathrm{SN}$ is created in Mininet [15]. The greedy algorithm presented in [8] is adapted to perform virtual to SN mapping through a node mapping. The Multi-Commodity Flow (MCF) [16] approach is used to perform link mapping. The bandwidth demand for virtual links configured on the SN as shown in Fig. 4 for edge, aggregation and core layer are $3 \mathrm{Mbps}, 4 \mathrm{Mbps}$ and 5 Mbps respectively.

The reception quality $\rho$ [17] is used for video quality measurements. The $\rho$ is defined as the ratio between download network throughput and video encoding rate (e.g., $D_{\text {thput }} / V_{\text {rate }}$ ). The $\rho$ quantify the impact of playback quality on the user's viewing experience during video streaming. The video has good reception quality if $\rho>1$ otherwise the video has poor reception quality. We also use the average switch and link resource utilization to measure the utilization of substrate resources in softwarized networks. The average switch utilization is defined as the average proportion of the total substrate switch capacity that is used during video streaming. The average link resource utilization is the total substrate link bandwidth capacity that is used at any given time during video streaming.

\section{B. Experimental Results and Discussion}

1) Average link resources utilization: Fig. 5a shows the average link resource utilization for our proposed approach. It is evident that, QoESoft achieves better performance in terms of link resources utilization. This is due to the fact that, resources are mapped efficiently based on the requirements of user's VN requests. The results indicate that as the number of $\mathrm{VN}$ requests increases the link utilization also increases. Fig. 5b indicates the average switch resource utilization for $500 \mathrm{VN}$ requests. Again, QoESoft achieves better results compared to when our approach is not used during video streaming. For example, at $350 \mathrm{VN}$ requests, QoESoft achieves 78\% of switch resources 


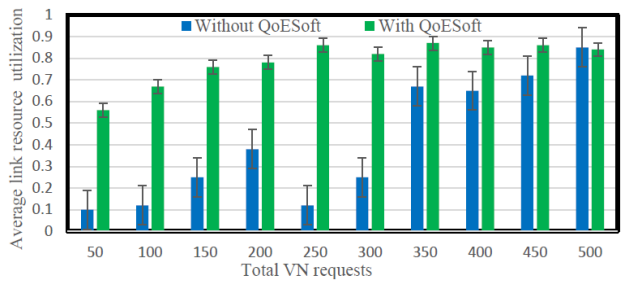

(a) Average link resources utilization

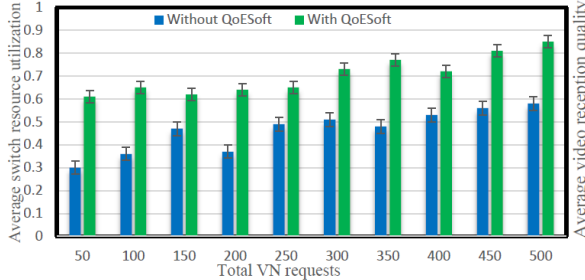

(b) Average switch resources utilization

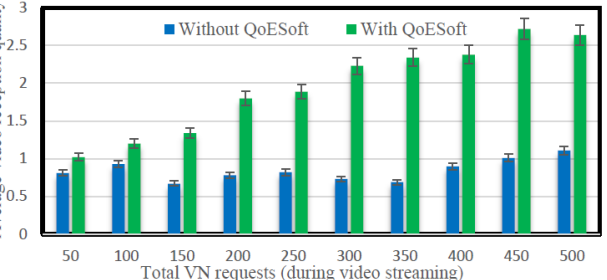

(c) Average video reception quality for $720 \mathrm{p}$ resolution

Fig. 5: Average resources utilization and video quality measurements

utilizations while when it is not used, only $48 \%$ resources are used utilized.

2) Video quality measurements: The results in Fig. 5c indicate that the video streaming experience using QoESoft is good for every $\mathrm{VN}$ requests because the quality reception for $720 \mathrm{p}$ resolution is $>1$. The video streaming experience for users is attributed by efficient utilization of resources for both link and switch in softwarized 5G systems. It is worth noting that, under/over-utilization of network resources is one of the factor that contributes to poor video quality and QoE-unfairness in DASH video streaming [9].

\section{CONClusion AND Future Work}

This paper is the first attempt towards developing QoE-aware resource management that consider both link and SDN switch resources in softwarized networks. We propose QoESoft, a 5G network softwarization approach for multimedia streaming services that performs an autonomic resource management in future softwarized 5G networks. Preliminary results indicate that, not only QoESoft achieves better performance in terms of network resources utilization but it also provides a good video streaming watching experience to the end users. Our future work will replicate this study and investigate resource management mechanisms for multimedia streaming services in $5 \mathrm{G}$ network slicing.

\section{ACKNOWLEDGEMENT}

This publication has emanated from research supported by the Irish Research Council (IRC) Postdoctoral Fellowship Programme 2019 - 2021 under the project: Management of Multimedia Services and Orchestration of Resources in 5G Networks using SDN and NFV (5GMOONS) and in part by a research grant from Science Foundation Ireland (SFI) and cofunded under the European Regional Development Fund under Grant Number 13/RC/2077 and Grant Number 12/RC/2289_P2.

\section{REFERENCES}

[1] A. Barakabitze, A. Arslan, M. Rashid, and H. Andrew, "5g network slicing using sdn and nfv: A survey of taxonomy, architectures and future challenges," Computer Networks, vol. 167, pp. 1-40, February 2020.

[2] A. A. Barakabitze, L. Sun, I.-H. Mkwawa, and E. Ifeachor, "A Novel QoE-Aware SDN-enabled, NFV-based Management Architecture for Future Multimedia Applications on 5G Systems," 8th International Conference on Quality of Multimedia Experience (QoMEX), June 2016.
[3] S. Sun, L. Gong, B. Rong, and K. Lu, "An intelligent sdn framework for 5g heterogeneous networks," IEEE Communications Magazine, vol. 53, no. 11, pp. 142-147, Nov 2015.

[4] R. Mijumbi, J. Serrat, J. Rubio-Loyola, N. Bouten, S. Latre, and F, "Dynamic resource management in sdn-based virtualized networks," IEEE International Workshop on Management of SDN and NFV Systems, Nov 2014.

[5] A. A. Barakabitze, L. Sun, I.-H. Mkwawa, and E. Ifeachor, "A Novel QoE-Centric SDN-based Multipath Routing Approach of Mutimedia Services over 5G Networks," in IEEE International Conference on Communications, Kansas City, MO, USA, May 2018.

[6] K. Kannan and S. Banerjee, "Compact tcam: Flow entry compaction in tcam for power aware sdn, in d. frey and $\mathrm{m}$. raynal, editors, distributed computing and networking, volume 7730 of lecture notes in computer science," vol. 7730, pp. 439-444, December 2013.

[7] I. Fajjari, N. Aitsaadi, G. Pujolle, and H. Zimmermann, "Vnr algorithm:a greedy approach for virtual networks reconfigurations," In GLOBECOM, pp. 1-6, May 2011.

[8] Y. Zhu and M. Ammar, "Algorithms for assigning substrate network resources to virtual network components," 25th IEEE International Conference on Computer Communications, pp. 1-12, 2006.

[9] A. Barakabitze, N. Barman, A. Ahmad, S. Zadtootaghaj, L. Sun, M. Martini, and Atzori, "Qoe management of multimedia services in future networks: A tutorial and survey," IEEE Communications Surveys and Tutorials, vol. 22, no. 1, pp. 526-565, December 2019.

[10] G. Elisavet, A. A. Barakabitze, L. Atzori, L. Sun, and V. Pilloni, "An SDN-approach for QoE Management of Multimedia Services using Resource Allocation," in IEEE International Conference Communications (ICC), Paris, France, May 2017, pp. 1-7.

[11] J. B. T. Feng and K. Wang, "Joint allocation and scheduling of network resource for multiple control applications in sdn," In Network Operations and Management Symposium (NOMS), pp. 1-7, May 2014.

[12] M. Varela, P. Zwickl, P. Reichl, M. Xie, and H. Schulzrinne, "From service level agreements (sla) to experience level agreements (ela): The challenges of selling qoe to the user," In proceedings of IEEE ICC QoEFI, June 2015.

[13] "GPAC:Multimedia Open Source Project," [Available at] :https://gpac.wp.imt.fr/mp4box/.

[14] A. Medina, A. Lakhina, I. Matta, and J. Byers, "Brite: An approach to universal topology generation," In Proceedings of the Ninth International Symposium in Modeling, Analysis and Simulation of Computer and Telecommunication Systems, MASCOTS, pp. 346-353, June 2001.

[15] R. L. S. de Oliveira, C. M. Schweitzer, A. A. Shinoda, and R. P. Ligia., "Using mininet for emulation and prototyping software-defined networks," IEEE Colombian Conference on Communications and Computing (COLCOM), pp. 1-6, June 2014.

[16] C. Barnhart, N. Krishnan, P. V. I. C. A. Floudas, and E. o. O. P. M. Pardalos, editors, "On QoE-awareness through Virtualized Probes in 5G Networks," 2016 IEEE 21st International Workshop on Computer Aided Modelling and Design of Communication Links and Networks (CAMAD), pp. 2354-2362, Oct 2009.

[17] A. A. Barakabitze, L. Sun, I.-H. Mkwawa, and E. Ifeachor, "QualitySDN: Improving Video Quality using MPTCP and Segment Routing in SDN/NFV ," IEEE Conference on Network Softwarization, May 2018. 